\title{
SOME PROPERTIES OF VECTOR MEASURES TAKING VALUES IN A TOPOLOGICAL VECTOR SPACE
}

\author{
EFSTATHIOS GIANNAKOULIAS
}

(Received 19 March 1986; revised 31 July 1986)

(Communicated by H. Lausch)

\begin{abstract}
In this paper we study some properties of vector measures with values in various topological vector spaces. As a matter of fact, we give a necessary condition implying the Pettis integrability of a function $f: S \rightarrow E$, where $S$ is a set and $E$ a locally convex space.

Furthermore, we prove an iff condition under which $(Q, E)$ has the Pettis property, for an algebra $Q$ and a sequentially complete topological vector space $E$.

An approximating theorem concerning vector measures taking values in a Fréchet space is also given.

1980 Mathematics subject classification (Amer. Math. Soc.): 38 B 05.
\end{abstract}

\section{Notations and terminology}

We denote by $S$ a non void set, $Q$ (resp. $\Sigma$ ) an algebra (resp. $\sigma$-algebra) of subsets of $S$ and $E$ a real Hausdorff locally convex space.

A function $\mu$ from the algebra $Q$ to $E$ is said to be a finitely additive vector measure (or simply a vector measure) if $\mu\left(A_{1} \cup A_{2}\right)=\mu\left(A_{1}\right)+\mu\left(A_{2}\right)$, whenever $A_{1}, A_{2}$ are disjoint members of $Q$.

If in addition $\mu\left(\cup_{n=1}^{\infty} A_{n}\right)=\sum_{n=1}^{\infty} \mu\left(A_{n}\right)$ for all sequences $\left(A_{n}\right)$ of pairwise disjoint members of $Q$ with $\bigcup_{n=1}^{\infty} A_{n} \in Q$ in the topology of $E$, then $\mu$ is called a $\sigma$-additive vector measure. We say that $\mu$ is strongly bounded ( $s$-bounded) iff $\lim _{n} \mu\left(A_{n}\right)=0$ for every sequence $\left(A_{n}\right)$ of mutually disjoint sets from $Q$.

(C) 1987 Australian Mathematical Society 0263-6115/87 \$A2.00+0.00 
If $\mu$ is an $E$-valued vector measure on $Q$ and $P$ a seminorm on $E$, we shall define the $P$-semivariation $P(\mu)$ by $P(\mu)(A)=\sup \left\{P\left(\sum_{j=1}^{n} a_{j} \mu\left(A_{j}\right)\right)\right\}, A \in Q$, where the supremum is taken over all disjoint sets $A_{1}, \ldots, A_{n}$ from $Q$ with $A=A_{1} \cup \cdots \cup A_{n}$ and all scalars $a_{1}, \ldots, a_{n}$ with $\left|a_{i}\right| \leqslant 1(i=1,2, \ldots, n)$. We say that the function $f: S \rightarrow E$ is weakly $\lambda$-summable with respect to measure $\lambda$ : $Q \rightarrow[0, \infty)$ if $\int_{A}\left|x^{\prime} f\right| d \lambda<\infty$ for all $x^{\prime} \in E^{\prime}, A$ in $Q . f$ is called $\lambda$-summable or Pettis integrable if it is weakly $\lambda$-summable for every $A$ in $Q$ and there exist an element $\int_{A} f d \lambda$, of $E$, such that

$$
x^{\prime} \int_{A} f d \lambda=\int_{A} x^{\prime} f d \lambda, \quad\left(x^{\prime} \in E^{\prime}\right)
$$

A locally convex space $E$ has the Bessaga-Petczynski property (shortly ( $B-P)$ property), if for every sequence $\left(x_{n}\right)$ from $E$ with $\sum_{n=1}^{\infty}\left|x^{\prime}\left(x_{n}\right)\right|<\infty$ for all $x^{\prime} \in E^{\prime}$, there exists $x \in E$ such that $x=\sum_{n=1}^{\infty} x_{n}$, where the series converges unconditionally.

Finally, a sequence $\left\{x_{n}\right\}$ in $E$ is a Schauder basis if every $x \in E$ has a unique representation in the form $x=\sum_{n=1}^{\infty} a_{n} x_{n}$, where $\left\{a_{n}\right\}$ is a sequence of scalars. For each $n \in \mathbf{N}$ the $n$th coefficient functional $f_{n}$ on $E$ is defined by $f_{n}(x)=a_{n}$, for all $x \in E$ and so $\mu(A)=\Sigma_{n \in \mathrm{N}} f_{n}(\mu(A)) x_{n}=\Sigma_{n \in \mathrm{N}} \mu_{n}(A) x_{n}, A$ in $Q$.

\section{On Pettis integral}

The purpose of this section is to extend a result of ([13], Theorem 1) to the case of vector measures which take values in a locally convex space $E$. This is given in 4. Theorem below.

1. Lemma ([9], Proposition 1). Let $\lambda: \Sigma \rightarrow[0,+\infty)$ be a measure and let $\mu$ : $\Sigma \rightarrow E$ be a s-bounded vector measure with $x^{\prime} \mu \ll \lambda$, for every $x^{\prime} \in E^{\prime}$. Then $\mu \ll \lambda$.

2. LeMMA. Let $f: S \rightarrow E$ be a vector function, $\nu: \Sigma \rightarrow E$ a vector measure and $(s, \Sigma, \lambda)$ a finite non negative measure space. We denote by $H$ the set $H=\left\{x^{\prime} \in E^{\prime}\right.$ : (i) $x^{\prime} f \in L_{1}(\lambda)$ and (ii) $x^{\prime} \circ \nu(A)=\int_{A} x^{\prime} f d \lambda A$ in $\left.\Sigma\right\}$. Then, for every $x^{\prime} \in H$, there exist a continuous seminorm $P_{x^{\prime}}$ on $E$ such that

$$
\int_{A}\left|x^{\prime} f\right| d \lambda \leqslant P_{x^{\prime}}(\nu)(A), \quad(A \text { in } \Sigma) .
$$


Proof. If $x^{\prime} \circ \nu=\mu$, then $\mu(A)=\int_{A} x^{\prime} f d \lambda$ and $\bigcup(\mu, A)=\int_{A}\left|x^{\prime} f\right| d \lambda$ (where $U(\mu, A) \leqslant\|\mu\|(A)$ (where $\|\mu\|$ denotes the semivariation of $\mu$ ), for if $A_{1}, \ldots, A_{n}$ are pairwise disjoint sets of $\Sigma$, then there exist complex numbers $a_{1}, \ldots, a_{n}$ with $\left|a_{i}\right|=1(i=1, \ldots, n)$ such that

$$
\sum_{i=1}^{n}\left|\mu\left(A_{i}\right)\right|=\left|\sum_{i=1}^{n} a_{i} \mu\left(A_{i}\right)\right| \leqslant\|\mu\|(A) .
$$

On the other hand,

$$
\left|\sum_{i=1}^{n} a_{i} \mu\left(A_{i}\right)\right|=\left|x^{\prime}\left(\sum_{i=1}^{n} a_{i} \nu\left(A_{i}\right)\right)\right| \leqslant\left|P_{x^{\prime}}\left(\sum_{i=1}^{n} a_{i} \nu\left(A_{i}\right)\right)\right| \leqslant P_{x^{\prime}}(\nu)(A)
$$

for some continuous seminorm $P_{x^{\prime}}$ on $E$, thus $\|\mu\|(A) \leqslant P_{x^{\prime}}(\nu)(A)$. The results now follows.

3. Lemma. Let $f: S \rightarrow E, \lambda: \Sigma \rightarrow[0,+\infty)$ a $\sigma$-additive measure and $\nu: \Sigma \rightarrow E$ a $\lambda$-continuous s-bounded vector measure. Then the set

$$
H=\left\{x^{\prime} \in E^{\prime}: \text { (i) } x^{\prime} f \in L_{1}(\lambda) \text { and (ii) } x^{\prime} \circ \nu(A)=\int_{A} x^{\prime} f d \lambda\right\}
$$

is weak* sequentially closed.

Proof. 2. Lemma implies that, for every $x^{\prime} \in H$, there exists a continuous seminorm $P_{x^{\prime}}$ on $E$ such that

$$
\int_{A}\left|x^{\prime} f\right| d \lambda \leqslant P_{x^{\prime}}(\nu)(A), \quad(A \text { in } \Sigma) .
$$

Suppose $\left\{x_{n}^{\prime}\right\}_{n=1}^{\infty}$ in $H$ and $x_{n}^{\prime}(x) \rightarrow x^{\prime}(x)$ (for all $x \in H$ ). Since $\nu \ll \lambda$ we have that $P_{x_{n}^{\prime}}(\nu) \ll \lambda, n=1,2, \ldots$.

In virtue of equality (1), we have $\lim _{\lambda(A) \rightarrow 0} \int_{A}\left|x_{n}^{\prime} f\right| d \lambda=0$ uniformly in $n \in \mathbf{N}$. Vitali's convergence theorem now says that $x^{\prime} f \in L_{1}(\lambda)$, hence

$$
\int_{A} x^{\prime} f d \lambda=\int_{A} \lim _{n}\left(x_{n}^{\prime} f\right) d \lambda=\lim _{n} \int_{A} x_{n}^{\prime} f d \lambda=\lim _{n} x_{n}^{\prime} \nu(A)=x^{\prime} \nu(A)
$$

and so $x^{\prime} \in H$.

4. TheOREM. Let $f: S \rightarrow E, \lambda: E \rightarrow[0,+\infty)$ a o-additive measure and $\nu$ : $\Sigma \rightarrow E$ a finite additive vector measure. Assume that:

(i) $H$ is a weak ${ }^{*}$ sequentially dense subset of $E$,

(ii) $x^{\prime} f \in L_{1}(\lambda)$ ( for all $\left.x^{\prime} \in H\right)$,

(iii) $x^{\prime} \nu(A)=\int_{A} x^{\prime} f d \lambda$ ( for all $A \in \Sigma$ and for all $x^{\prime} \in H$ ).

Then $f$ is Pettis $\lambda$-integrable and

$$
\nu(A)=(P) \int_{A} f d \lambda \quad(A \in \Sigma)
$$


Proof. Assumption (iii) implies $x^{\prime} \nu \ll \lambda$, for every $x^{\prime} \in H$. Since $H$ is a weak* sequentially dense subset of $E^{\prime}$, we have that $x^{\prime} \nu \ll \lambda$, for every $x^{\prime} \in E^{\prime}$. Hence, $x^{\prime} \nu$ is $\sigma$-additive for every $x^{\prime} \in E^{\prime}$ and thus $\nu$ is $\sigma$-additive by the Orlicz-Pettis theorem. Since $\Sigma$ is a $\sigma$-algebra $\nu$ is also a $s$-bounded vector measure and from 1 . Lemma we have that $\nu \ll \lambda$. 3. Lemma now implies that $H$ is weak* sequentially closed and so $H=E^{\prime}$. Hence we have that

$$
x^{\prime} \nu(A)=\int_{A} x^{\prime} f d \lambda, \quad \text { for every } x^{\prime} \in E^{\prime},
$$

which proves the assertion.

\section{The Pettis property}

If $Q$ is a Boolean algebra and $X$ is a Banach space, we shall say that the pair $(Q, X)$ has the Pettis property if every weakly countably additive set function $\mu$ : $Q \rightarrow X$ is $\sigma$-additive. It is proved by [7] that a pair $(Q, X)$ has the Pettis property, for every algebra $Q$, if and only if $X \nsupseteq c_{0}$. A generalization of this is 5 . Theorem below for the case of a sequentially complete topological vector space.

5. THEOREM. Let $Q$ be an algebra of sets and let $E$ be a $q$ sequentially complete topological vector space. Then the following propositions are equivalent:

(i) $(Q, E)$ has the Pettis property,

(ii) $E$ has the ( $B-P)$-property.

Proof. (i) $\Rightarrow$ (i). We suppose that $E$ does not have the (B-P)-property. Then, there exists a sequence $\left(x_{n}\right)$ on $E$ such that $\sum_{n=1}^{\infty}\left|x^{\prime}\left(x_{n}\right)\right|<\infty$, for every $x^{\prime} \in E^{\prime}$ and the series $\sum_{n=1}^{\infty} x_{n}$ does not converge. From ([14], Theorem 4) now we have that $c_{0}$ is isomorphic to a subspace of $E$. But there exists a vector set function $\mu$ : $Q \rightarrow c_{0}$ which is weakly $\sigma$-additive but not $\sigma$-additive ([11], example 7).

(ii) $\Rightarrow$ (i). Let $\mu: Q \rightarrow E$ be weakly $\sigma$-additive and $\left(A_{n}\right)$ a disjoint sequence of sets in $Q$ with $\bigcup_{n=1}^{\infty} A_{n} \in Q$. Then $x^{\prime} \mu\left(\bigcup_{n=1}^{\infty} A_{n}\right)=\sum_{n=1}^{\infty} x^{\prime} \mu\left(A_{n}\right)$ (the series converges unconditionally) for all $x^{\prime} \in E^{\prime}$. Hence $\sum_{n=1}^{\infty}\left|x^{\prime} \mu\left(A_{n}\right)\right|<\infty$. Since $E$ has the (B-P)-property, the series $\sum_{n=1}^{\infty} \mu\left(A_{n}\right)$ converges unconditionally and so, for $x^{\prime} \in E^{\prime}$, we have $x^{\prime}\left(\sum_{n=1}^{\infty} \mu\left(A_{n}\right)\right)=\sum_{n=1}^{\infty} x^{\prime} \mu\left(A_{n}\right)=x^{\prime} \mu\left(\cup_{n=1}^{\infty} A_{n}\right)$ and $\sum_{n=1}^{\infty} \mu\left(A_{n}\right)=\mu\left(\bigcup_{n=1}^{\infty} A_{n}\right)$.

However, in the case of locally convex space with a Schauder basis, the $\sigma$-additivity of the measure, with respect to the topology, is equivalent to the $\sigma$-additivity of the real measures $\mu_{n}=f_{n} \circ \mu$, where the $f_{n}$ are the functionals 
associated to the basis. As a matter of fact, one obtains

6. Proposition ([8], Proposition 2). Let E be a locally convex space with a Schauder basis $\left(x_{n}, f_{n}\right)$ and $\mu: Q \rightarrow E$ a vector measure. Then the following are equivalent:

(i) $\mu$ is a $\sigma$-additive,

(ii) $\mu_{n}$ is $\sigma$-additive, $(n \in \mathbf{N})$.

\section{An approximation theorem for vector measures}

Let $E$ be a Fréchet space, $\mathscr{U}$ a fundamental system of neighbourhoods of zero in $E$ (consisting of closed and absolutely convex sets) and $\left(P_{v}\right)_{v \in \mathscr{q}}$ the family of the Minkowski functionals.

The function $f: S \rightarrow E$ is called $\lambda$-integrable with respect to the measure $\lambda$ : $\Sigma \rightarrow[0,+\infty)$, if $f$ is strongly measurable and, for every $v \in \mathscr{U}$, we have $\int_{s} P_{v}(f) d \lambda<\infty$. We denote $L^{1}(S, \lambda, E)$ the quotient space $\mathscr{L}^{1}(S, \lambda, E) / n$, where $\mathscr{L}^{1}(S, \lambda, E)$ is the space of all $\lambda$-integrable functions $f: L S \rightarrow E$ and $n=\left\{f \in \mathscr{L}^{1}(S, \lambda, E)\right.$ such that $\left.q_{v}(F)=0, v \in \mathscr{U}\right\}$. Note that $L^{1}(S, \lambda, E)$ is a Fréchet space with the topology defined by the family of seminorms $q_{v}, v \in \mathscr{U}$, where $q_{v}(f)=\int_{s} P_{v}(f) d \lambda$. Let $\mu: \Sigma \rightarrow E$ be a vector measure. We say that $\mu$ is of bounded variation if

$$
V(\mu, v)(S)=\sup \left\{\sum_{i=1}^{n} P_{v}\left(\mu\left(S_{i}\right)\right), S_{i} \in \Sigma, S_{i} \subset S \text { disjoint }\right\}<\infty
$$

for every $v \in \mathscr{U}$.

We define the measure $\lambda_{f}(S)=\int_{s} f d \lambda$, for all $f \in L^{1}(S, \lambda, E)$, satisfying $V\left(\lambda_{f}, v\right)(S)=\int_{s} P_{v}(f) d \lambda$. It is a measure of bounded variation and satisfies ([3], page 372$)$

$$
P_{v}\left(\lambda_{f}(S)\right) \leqslant \int_{s} P_{v}(f) d \lambda
$$

We are able to state and prove the second main theorem.

7. THEOREM. Let $(S, Q, \lambda)$ be a finite (positive) measure space, $E$ a Fréchet space with the Radon-Nikodym property and $\mu: Q \rightarrow E$ an additive vector measure of bounded variation with $\mu \ll \lambda$. Then, there exist a sequence $\left\{\phi_{n}\right\}$ of simple functions $\phi_{n}: S \rightarrow$ E such that

$$
P_{v}\left(\int_{A} \phi_{n} d \lambda-\mu(A)\right) \underset{n}{\rightarrow} 0
$$

for every $A \in Q$ and for all $v \in \mathscr{U}$. 
Proof. By Stone's theorem ([5], Theorem 1) there exists a totally disconnected compact Hausdorff space $K$, for which the algebra $\hat{Q}$ of all open-closed subsets of $K$ is isomorphic to the algebra $Q$. Let $\phi$ be the above isomorphism. We define $\hat{\mu}$ : $\hat{Q} \rightarrow E$ by $\mu(\phi(A)):=\mu(A)$ and $\hat{\lambda}: \hat{Q} \rightarrow[0,+\infty)$ by $\hat{\lambda}(\phi(A)):=\lambda(A) . \hat{\lambda}$ is regular ([1], Theorem 2); therefore, $\hat{\lambda}$ is $\sigma$-additive ([6], Theorem 13, page 138), Hahn's extension theorem now implies that exists a unique extension of $\hat{\lambda}$ (denoted also by $\hat{\lambda}$ ) to the $\sigma$-algebra $\Sigma_{0}$ generated by $\hat{Q}$. We consider the standard metric on $\Sigma_{0}, d\left(E_{1}, E_{2}\right)=\hat{\lambda}\left(E \Delta E_{2}\right)$ and we denote the resulting metric space by $\Sigma_{0}(\hat{\Lambda})$. Recall that $\hat{Q}$ is then a dense subset of $\Sigma_{0}(\hat{\lambda})$ ([10], [13], Theorem D). Therefore, the function $\hat{\mu}: Q \rightarrow \Sigma_{0}(\hat{\lambda}) \rightarrow E$ is continuous (since $\mu \ll \lambda$ implies $\hat{\mu} \ll \hat{\lambda})$ and it has an extension, denoted also by $\hat{\mu}, \hat{\mu}: \Sigma_{0}(\hat{\lambda}) \rightarrow E$. Now, from Radon-Nikodym's theorem, there exists $\hat{f} \in L_{1}\left(\hat{\lambda}, \Sigma_{0}, E\right)$ such that

$$
\hat{\mu}(A)=\int_{A} \hat{f} d \hat{\lambda} \quad\left(\text { for all } A \in \Sigma_{0}\right) .
$$

(This is denoted by $\hat{\mu}=\hat{f} \hat{\lambda}$.) Hence there exists a sequence $\hat{\phi}_{n}$ of simple functions converging to the function $\hat{f}$, that is,

$$
q_{v}\left(\hat{\phi}_{n}-\hat{f}\right)=\int_{s} P_{v}\left(\hat{\phi}_{n}-\hat{f}\right) d \hat{\lambda} \rightarrow 0 \quad \text { for all } v \in \mathscr{U} .
$$

We also have that

$$
\hat{\lambda}_{(\hat{\phi}-\hat{\jmath})}(S)=\int_{s}\left(\hat{\phi}_{n}-\hat{f}\right) d \hat{\lambda}=\left(\hat{\phi}_{n}-\hat{f}\right) \hat{\lambda}(S)
$$

is a vector measure of bounded variation.

From (1) and (2) we obtain

$$
P_{v}\left(\hat{\lambda}_{\hat{\phi}_{n}-\hat{f}}(S)\right)=P_{v}\left(\int_{s}\left(\hat{\phi}_{n}-\hat{f}\right) d \hat{\lambda}\right) \leqslant \int_{s} P_{v}\left(\hat{\phi}_{n}-\hat{f}\right) d \hat{\lambda} .
$$

Hence $P_{v}\left(\hat{\lambda}_{\hat{\phi}_{n}-j}(A)\right) \rightarrow 0$, for all $A \in Q$, therefore $P_{v}\left[\left(\hat{\phi}_{n}-\hat{f}\right) \hat{\lambda}(A)\right] \rightarrow 0$. So $P_{v}\left[\hat{\phi}_{n} \hat{\lambda}(A)-\hat{f} \hat{\lambda}(A)\right] \rightarrow 0$ and

$$
P_{v}\left[\phi_{n} \lambda(A-\mu)(A)\right] \underset{n}{\rightarrow} 0, \quad \text { for all } A \in Q \text { and } v \in \mathscr{U} .
$$

\section{Acknowledgement}

The author is indebted to the referee for his suggestions. 


\section{References}

[1] R. Alo and A. Korvin, 'Some approximation theorems for vector measures', Rev. Roumaine Math. Pures Appl. 23 (1979), 1289-1295.

[2] C. Bessaga and A. Pelczyński, 'On bases and unconditional convergence of series in Banach spaces', Studia Math. 18 (1958), 151-164.

[3] H. Chi, 'A geometric characterization of Fréchet spaces with the Radon-Nikodym property', Proc. Amer. Math. Soc. 48 (1975), 371-380.

[4] J. Diestel and J. J. Uhl, Vector measures (Math. Surveys 15, Amer. Math. Soc., Providence, R.I., 1977).

[5] N. Dinculeanu, Vector measures (Veb. Deutsher Verlag der wissenschaften, Berlin, 1966).

[6] N. Dunford and J. Schwartz, Linear operators, Part I (Interscience, New York, 1958).

[7] B. Faires, Grothendieck spaces and vector measures ( $\mathrm{Ph}$. D. Thesis, Kent State University, 1974).

[8] E. Giannakoulias, 'The Bessaga-Pelczynski property and strongly bounded measures', Bull. Soc. Math. Gréce 24 (1983).

[9] E. Giannakoulias, 'Absolute continuity and decomposability of vector measures taking values in a locally convex space with basis', to appear.

[10] P. Halmos, Measure theory (Van Nostrand, 1950).

[11] J. Hofman-Jørgensen, 'Vector measures', Math. Scand. 28 (1971), 5-32.

[12] J. Kluvanek and G. Knowles, Vector measures and control systems (Notas Mat. 58 (1976), North-Holland).

[13] Z. Lipecki and $\mathrm{K}$. Musial, 'On the Radon-Nikodym derivative of a measure taking value in a Banach space with basis', Rev. Roumaine Math. Pures Appl. 23 (1978), 911-915.

[14] D. Tumarkin, 'On locally convex spaces with basis', Dokl. Akad. Nauk SSSR 11 (1970), $1672-1675$.

\section{Department of Mathmatics}

Section of Mathematical Analysis and its Applications

Athens University

Panepistemiopolis, 15781 Athens

Greece 